\title{
1 Accelerated DNA evolution in rats is driven by differential methylation
}

\section{2 in sperm}

3 Xiao-Hui Liu, ${ }^{1,2, *}$ Jin-Min Lian,,${ }^{3, *}$ Fei Ling, ${ }^{4,}{ }^{*}$ Ning Li,,${ }^{1,2}$ * Da-Wei Wang,, 2, * Ying Song,, , , *

4 Qi-Ye Li, ${ }^{3,5}$ Ya-Bin Jin, ${ }^{4}$ Zhi-Yong Feng, ${ }^{6}$ Lin Cong, ${ }^{7}$ Dan-Dan Yao, ${ }^{6}$ and Jing-Jing Sui ${ }^{6}$

$5 \quad{ }^{1}$ State Key Laboratory for Biology of Plant Diseases and Insect Pests, ${ }^{2}$ Key Laboratory of Weed

6 and Rodent Biology and Management, Institute of Plant Protection, Chinese Academy of

7 Agricultural Sciences, Beijing 100193, China; ${ }^{3}$ China National GeneBank, BGI-Shenzhen,

8 Shenzhen, Guangdong 518083, China; ${ }^{4}$ School of Bioscience and Bioengineering, South China

9 University of Technology, Guangzhou, Guangdong 510006, China; ${ }^{5}$ Center for GeoGenetics,

10 Natural History Museum of Denmark, University of Copenhagen, Øster Voldgade 5-7,

11 Copenhagen 1350, Denmark; ${ }^{6}$ Guangdong Provincial Key Laboratory of High Technology for

12 Plant Protection, Plant Protection Research Institute, Guangdong Academy of Agricultural

13 Sciences, Guangzhou, Guangdong 510640, China; ${ }^{7}$ Plant Protection Institute, Heilongjiang

14 Academy of Agricultural Sciences, Harbin, Heilongjiang 150086, China.

15 "These authors contributed equally to this work.

17 Correspondence: Professor Xiao-Hui Liu, Institute of Plant Protection, Chinese Academy of

18 Agricultural Sciences, West Yuanmingyuan Road 2\#, Hai Dian District, Beijing 100193, China.

19 Tel: 86-10-62818873; Email: liuxiaohui@caas.cn

21 Running title: DNA evolution driven by differential methylation

23 Keywords: transgenerational epigenetic inheritance; DNA evolution; methylome; genome; sperm;

24 Rattus norvegicus. 


\section{Summary}

26 Lamarckian inheritance has been largely discredited until the recent discovery of

27 transgenerational epigenetic inheritance. However, transgenerational epigenetic inheritance is still

28 under debate for unable to rule out DNA sequence changes as the underlying cause for

29 heritability. Here, through profiling of the sperm methylomes and genomes of two recently

30 diverged rat subspecies, we analyzed the relationship between epigenetic variation and DNA

31 variation, and their relative contribution to evolution of species. We found that only epigenetic

32 markers located in differentially methylated regions (DMRs) between subspecies, but not within

33 subspecies, can be stably and effectively passed through generations. DMRs in response to both

34 random and stable environmental difference show increased nucleotide diversity, and we

35 demonstrated that it is variance of methylation level but not deamination caused by methylation

36 driving increasing of nucleotide diversity in DMRs, indicating strong relationship between

37 environment-associated changes of chromatin accessibility and increased nucleotide diversity.

38 Further, we detected that accelerated fixation of DNA variants occur only in inter-subspecies

39 DMRs in response to stable environmental difference but not intra-subspecies DMRs in response

40 to random environmental difference or non-DMRs, indicating that this process is possibly driven

41 by environment-associated fixation of divergent methylation status. Our results thus establish a

42 bridge between Lamarckian inheritance and Darwinian selection. 
44 DNA variation passed stably from parent to offspring is the traditional mechanism underlying

45 trait heritability, and provides the basis for Darwinian selection. In contrast, transgenerational

46 inheritance of epigenetic variation has recently been proposed as a form of Lamarckian acquired

47 inheritance, where species adapt to the changing environment without an accompanying DNA

48 sequence change (Van Soom et al. 2014). Transgenerational epigenetic inheritance has been

49 controversial as it was previously thought that the epigenome is fully erased and reestablished

50 between generations in order for appropriate cellular development and differentiation to occur in

51 mammals (Daxinger and Whitelaw 2012; Franklin and Mansuy 2010; Heard and Martienssen

52 2014). In recent years, Lamarckian acquired inheritance has gained increasing support due to the

53 findings of transgenerational transmission of epigenetic status in a number of species (Daxinger

54 and Whitelaw 2012; Franklin and Mansuy 2010; Heard and Martienssen 2014; Lim and Brunet

55 2013; Van Soom et al. 2014). However, transgenerational epigenetic inheritance and Lamarckian

56 acquired inheritance is still under debate for unable to rule out DNA sequence changes as the

57 underlying cause for heritability (Heard and Martienssen 2014; Lim and Brunet 2013).

58 Distinguishing the relative contributions of epigenetic changes and DNA variation to phenotypic

59 variation and determining the potential for epigenetics to impact DNA evolution are keys to

60 resolving these questions (Boffelli and Martin 2012; Heard and Martienssen 2014; Lim and

61 Brunet 2013). The mutation rate at $\mathrm{CpG}$ sites is influenced by methylation status (Fryxell and

62 Moon 2005; Mugal and Ellegren 2011; Xia et al. 2012; Zhao and Jiang 2007), making

63 methylation a plausible mechanism by which the epigenome influences heritable traits through

64 changes to the underlying DNA sequence. However, the relationship between epigenetic variation

65 and DNA variation, their relative contribution to phenotypic divergence, and relative impact on

66 the evolution of species are far from clear (Heard and Martienssen 2014; Lim and Brunet 2013).

67 The Norway rat originated in South China 1.2-1.6 million years ago and spread throughout

68 the rest of the world with humans (Song et al. 2014; Wu and Wang 2012). The split of two

69 subspecies Rattus norvegicus caraco (Rnc) and Rattus norvegicus norvegicus (Rnn), distributed 
70 in North and South China respectively, has been supported by both morphological data and

71 mitochondrial DNA analysis (Song et al. 2014). R. n. norvegicus breed year round while R. $n$.

72 caraco has restricted breeding during the winter (Wang et al. 2011). Intriguingly, we observed

73 that the latter can breed year round when reared in proper room conditions, indicating the

74 reproductive activity of the Norway rat is very sensitive to environmental change. The plasticity

75 of this trait may indicate that it is an example of Lamarckian inheritance. Additionally,

76 reproductive behavior involves the coordinated expression of a cohort of genes, making it likely

77 that epigenetic inheritance could play a role. Thus, these two subspecies provide a nice model to

78 explore the relationship between epigenetic variation and DNA variation and their contribution to

79 species divergence. In this study, we analyzed DNA sequence and methylation variation in sperm

80 to illustrate the relationship between transgenerational epigenetic inheritance and DNA evolution

81 in the divergence of $R$. n. norvegicus and R. n. caraco. We found that all kinds of

82 environment-associated methylation differences can lead to increasing of nucleotide diversity,

83 and fixation of methylation status can further lead to accelerated fixation of DNA variants. These

84 results establish a bridge between Lamarckian acquired inheritance and Darwinian selection.

\section{Results}

\section{DNA evolution is associated with environment-dependent methylation pattern}

88 Two individuals were selected from each Norway rat subspecies R. n. caraco and R. $n$.

89 norvegicus, located in Harbin City $\left(126^{\circ} 32^{\prime} \mathrm{E}, 45^{\circ} 48^{\prime} \mathrm{N}\right)$ and Zhanjiang City $\left(110^{\circ} 21^{\prime} \mathrm{E}, 21^{\circ} 16^{\prime} \mathrm{N}\right)$,

90 respectively. Both the genome and sperm methylome were sequenced for each sample, and each

91 methylome was proofread using the corresponding genome sequence of this individual. On

92 average, we generated more than $20 \times$ coverage of genome sequence for each sample, and over $89 \%$

93 of the non-gap genome was covered by more than one read (Supplemental Table 1). The basic

94 BS-seq data analysis was conducted using a custom pipeline validated in several previous

95 projects (Bonasio et al. 2012; Shao et al. 2014). Each methylome was proofread by genome 
96 sequence of this individual. About 22 million CpGs (90\% of all rat CpGs) were covered by at

97 least one read. The average read coverage for CpGs is at least $23 \times$ per sample with an overall

98 methylation level of $76 \%$ for all CpG sites (Supplemental Table 1). The non-CpG sites were not

99 significantly methylated in any of the datasets.

100 In total, we obtained 1,859 inter-subspecies DMRs (Differentially methylated regions) with

101 a total sequence length of 2.60 Mb (Supplemental Table 2), 2,669 intra-Rnn DMRs with a total

102 length of 2.67 Mb (Supplemental Table 3), and 3,461 intra-Rnc DMRs with a length of 3.46 Mb

103 (Supplemental Table 4). We found that in $87.74 \%$ of inter-subspecies DMRs methylation levels

104 are the same between individuals within each subspecies (Supplemental Fig. 1A), indicating the

105 methylation statuses in the inter-subspecies DMRs are subspecies specific and retained across

106 generations. Although the total sequence length of each DMR set covers only one thousandth of

107 the genome, each set of DMRs was distributed evenly throughout the genome (Supplemental Fig.

108 1B). Genome sequences were classified as DMRs or non-DMRs for further analysis.

109 As Figure 1 illustrates, phylogenetic analysis of sperm methylomes, genome-wide single

110 nucleotide variants (SNVs), DMR sequences and sampled non-DMR sequence (the first sampling

111 method) all support the divergence between the two subspecies, but show different tree topologies.

112 The topology of the tree built using DNA sequences in inter-subspecies DMRs is similar to the

113 topology of the clustering tree constructed using methylation patterns (Fig. 1A, 1B and 1D) but

114 not sampled non-DMR sequences or genome-wide SNVs, and the topology of the tree built using

115 sampled non-DMR sequences is similar to the topology of the tree constructed using

116 genome-wide SNVs (Fig. 1C and 1E), indicating a possible correlation between methylation

117 pattern and DNA divergence. Compared to sampled non-DMR sequence with equal length and

118 GC content, DNA sequences in inter-subspecies DMRs show increased mean distance between

119 subspecies (independent t test: $p=0$; Fig.1; Supplemental Fig. 2A), but decreased mean distance

120 within subspecies (independent t test: $p<0.01$; Fig.1; Supplemental Fig. 2B and 2C), indicating 
121 that methylation variation in inter-subspecies DMRs may promote species divergence while

122 decreases the within-species DNA divergence.
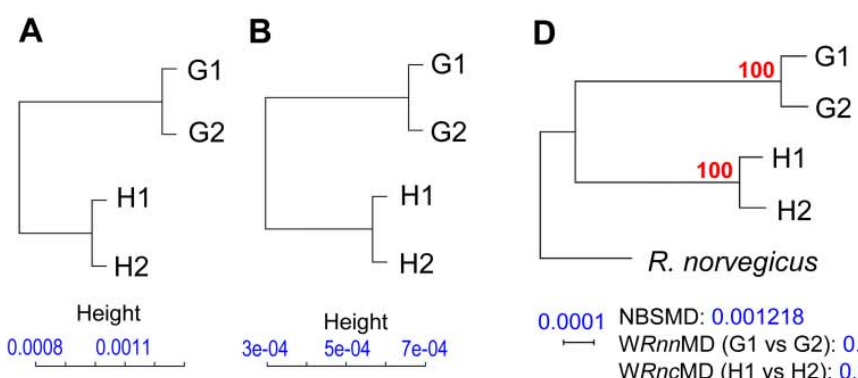

0.0001 NBSMD: 0.001218 WRnnMD (G1 vs G2): 0.000171
WRncMD (H1 vs H2): 0.000159

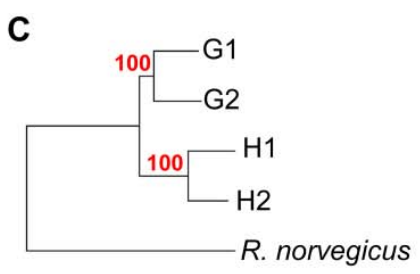

$\stackrel{0.1}{.}$

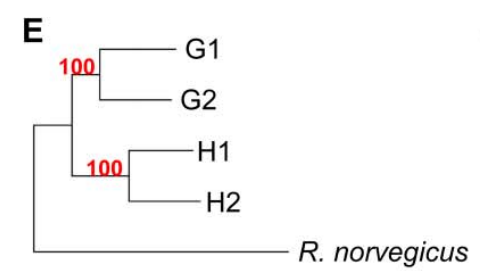

0.00005 NBSMD: 0.000092

WRnnMD (G1 vs G2): 0.000184 WRncMD ( $\mathrm{H} 1$ vs $\mathrm{H} 2)$ : 0.000169

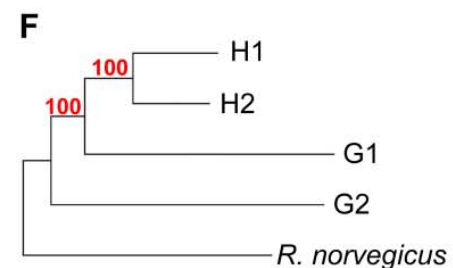

0.00005 NBSMD: 0.000056 WRnnMD (G1 vs G2): 0.000841 WRncMD (H1 vs $\mathrm{H} 2): 0.000238$

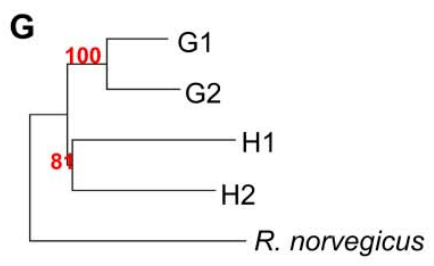

0.00005 NBSMD: 0.000077 WRnnMD (G1 vs G2): 0.000230 WRncMD ( $\mathrm{H} 1$ vs $\mathrm{H} 2)$ : 0.000527

123

124 Figure 1. Evolution patterns of inter-subspecies DMRs, intra-subspecies DMRs and non-DMRs.

125 Rat G1 and G2 are two individuals of $R$. n. norvegicus, and Rat $\mathrm{H} 1$ and $\mathrm{H} 2$ are two individuals of

126 R. n. caraco. Non-DMRs were sampled using the first method with equal length and similar GC

127 content as inter-subspecies DMRs. Except methylation trees, all trees were constructed using the

128 Neighbor-Joining method in the PHYLIP software package. NBSMD: Net between subspecies

129 mean distance. WRnnMD: Within Rnn mean distance. WRncMD: Within Rnc mean distance.

130 (A) Methylation clustering tree for the 4 rats. Methylation levels are calculated using genes. (B)

131 Methylation clustering tree for the 4 rats. Methylation levels are calculated using 10kb sliding

132 windows. (C) Phylogenetic tree constructed using genome-wide SNVs. (D) The phylogenetic tree

133 constructed using combined inter-subspecies DMR sequences. $(E)$ The phylogenetic tree

134 constructed using a set of sampled non-DMR sequences. $(F)$ The phylogenetic tree constructed

135 using combined intra-Rnn DMR sequences. $(G)$ The phylogenetic tree constructed using

136 combined intra-Rnc DMR sequences. 
137 In comparison, both phylogenetic trees built with the DNA sequences in intra-subspecies

138 DMRs have decreased mean distances between subspecies than both inter-subspecies DMRs and

139 non-DMRs (Fig. 1F and 1G). The phylogenetic tree built using four sequences in intra-Rnn

140 DMRs shows increased distance within Rnn but not Rnc (Fig. 1F), and the phylogenetic tree built

141 with the four sequences in intra-Rnc DMRs shows increased distance within Rnc but not Rnn (Fig.

$1421 \mathrm{G})$. This indicates that methylation variation and its related DNA variation in intra-subspecies

143 DMRs are not associated with species divergence.

144 We calculated Tajima's D to test for departure from neutrality in these regions. Tajima's D

145 is $1.678528,-0.393469$ and -0.277028 in combined inter-subspecies, intra-Rnn and intra-Rnc

146 DMR sequences, respectively (Fig. 2). In comparison, the mean Tajima's D in 1,000 sampled

147 non-DMR sequences is $0.002920 \pm 0.099323(\mathrm{SD})$, with a maximum value of 0.453000 , which is

148 significantly lower than in inter-subspecies DMRs (independent $t$ test: $p=0$; Fig. 2). The

149 distribution of Tajima's D in non-DMRs is normal with a median value of 0 , indicating the

150 sampled non-DMRs are a set of random neutral sequences. The increased Tajima's D value in the

151 inter-subspecies DMRs indicates strong selection and existence of a larger proportion of fixed or

152 fixing SNVs between subspecies in inter-subspecies DMRs. The significantly negative Tajima's

153 D in both sets of intra-subspecies DMRs indicates an excess of random substitutions (Fig. 2). 


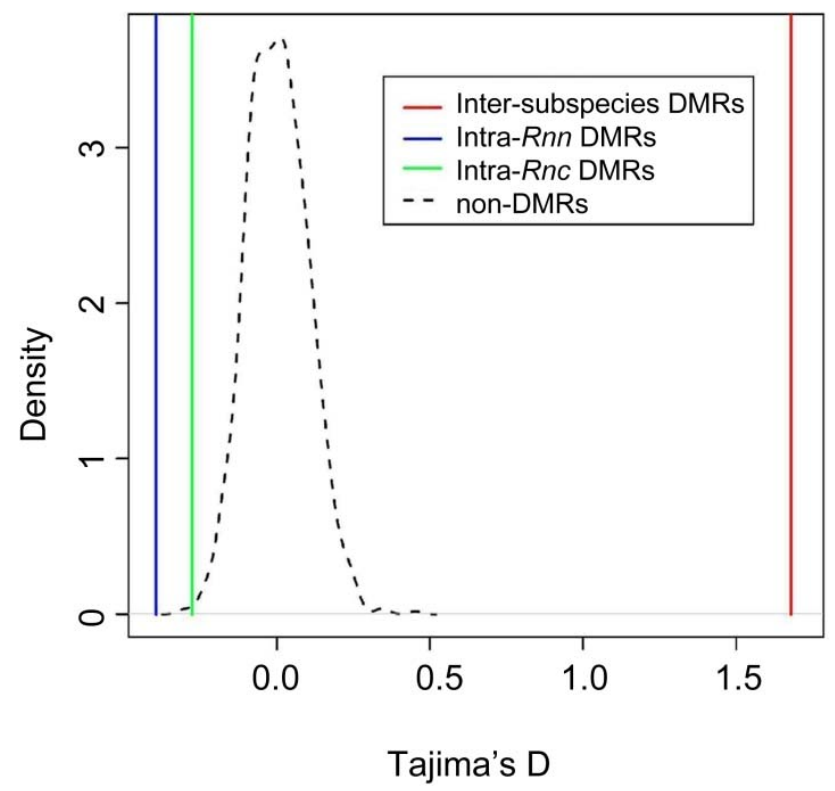

155 Figure 2. Comparison of Tajima's D using the second sampling method.

We wanted to characterize SNVs that are fixed or fixing in one of the subspecies, which we 158 will refer to as subspecies specific SNVs (SS-SNVs) (Supplemental Fig. 3), To accomplish this 159 we compared the DNA divergence level between DMRs and non-DMRs by calculating the ratio 160 of SS-SNVs (RSS, SS-SNVs were identified under $p$ value of 0.05 and 0.01 , respectively) using 16110 additional genome sequences ( 5 for each subspecies, kindly provided by the Kunming Institute 162 of Zoology, CAS). Inter-subspecies DMRs have significantly higher RSSs than non-DMRs 163 (Chi-Square test; DMRs: $42.95 \%$ at significant level $<0.05$ of SS-SNVs statistic, 30.88\% < 0.01; non-DMRs: $14.87 \%<0.05,8.87 \%<0.01$; Table $1 ; \mathrm{x}^{2}=1587.585 \mathrm{df}=1, p<2.2 \mathrm{e}-16$, under the $\mathrm{p}$ value of $0.05 ; \mathrm{x}^{2}=1786.16, \mathrm{df}=1, p<2.2 \mathrm{e}-16$, under the $p$ value of 0.01 ). These results further confirmed the accelerated DNA evolution in inter-subspecies DMRs. 
Table 1. Population test of RSS

\begin{tabular}{llcccc}
\hline Location & SNVs & SS-SNVs identified & SNVs covered & SS-SNVs identified & SS-SNVs identified \\
& identified in 4 & in 4 genomes (RSS) & in 14 genomes & in 14 genomes $(p$ & in 14 genomes $(p<$ \\
& genomes & & & $<0.01)(\mathrm{RSS})$ & $0.05)(\mathrm{RSS})$ \\
& & & & & \\
\hline Genome & $12,669,132$ & $2,722,049$ & $3,236,117$ & 28,7893 & 482,520 \\
non-DMRs & $12,648,498$ & $2,711,624(21.44 \%)$ & $3,231,817$ & $286,565(8.87 \%)$ & $480,673(14.87 \%)$ \\
DMRs & 20,634 & $10,425(50.52 \%)$ & 4,300 & $1,328(30.88 \%)$ & $1,847(42.95 \%)$ \\
& & & & & \\
\hline
\end{tabular}

173 Nucleotide diversity is promoted by variation of methylation level rather than methylation

\section{4 level itself.}

175 To explore the relationship between DNA methylation variation and substitution rate, we

176 compared nucleotide diversity $(\pi)$ between DMRs and non-DMRs. Using the second sample

177 method of non-DMRs, we found that the distribution pattern of $\pi$ was positively skewed in both

178 inter- and intra-subspecies DMRs by some higher $\pi$ values, but was normal in both inter- and

179 intra-subspecies non-DMRs (Supplemental Fig. 4). Mann-Whitney tests revealed $\pi$ in both inter-

180 and intra-subspecies DMRs was significantly higher than that of non-DMRs $(p<0.00001$; Fig.

1813 A, Supplemental Table 5). We also calculated $\pi$ in the combined DMRs of the whole genome

182 for the inter-subspecies, intra-Rnn, intra-Rnc DMRs and sampled non-DMRs (by the second

183 sampling method), respectively. The distribution of $\pi$ in the 1,000 sampled combined non-DMRs

184 is normal with a small standard deviation $(0.001844 \pm 0.000053$; Fig. 3B), indicating DNA

185 variation patterns in non-DMRs are similar and random. The value of $\pi$ in the combined

186 inter-subspecies, intra-Rnn and intra-Rnc DMR sets is $0.00343,0.003055$ and 0.0026 ,

187 respectively, which are significantly higher than both the average and the maximum $\pi$ value of 
188 sampled non-DMRs (independent t test: $p=0$; Fig. 3B), indicating extremely high levels of DNA

189 variation within DMRs. We then stratified DMRs by methylation level and found that DMRs

190 have a significantly higher $\pi$ than non-DMRs irrespective of the methylation level

191 (Mann-Whitney test: $p<0.00001$; Fig. 4, Supplemental Table 6), suggesting that the nucleotide

192 diversity in DMRs could be determined by variation of methylation level rather than methylation

193 level itself.

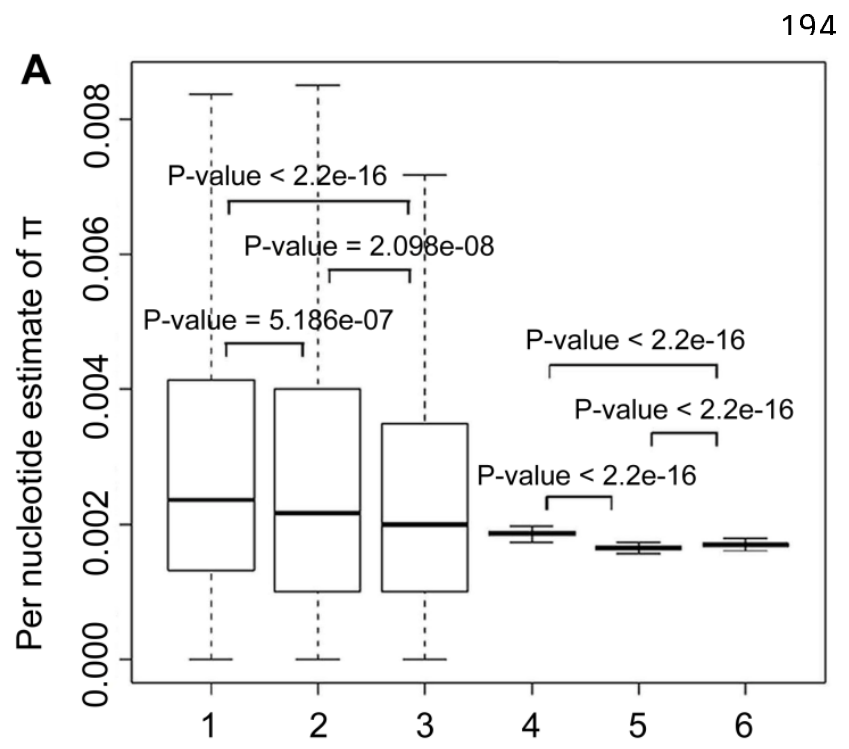

Figure 3. DMRs in response to both random and stable environmental difference show increased nucleotide diversity. $(A)$ Comparison of $\pi$ using second sampling method. $\pi$ of inter-subspecies DMRs or non-DMRs are calculated using 4 individuals, and $\pi$ of intra-subspecies DMRs or non-DMRs are calculated using 2 individuals

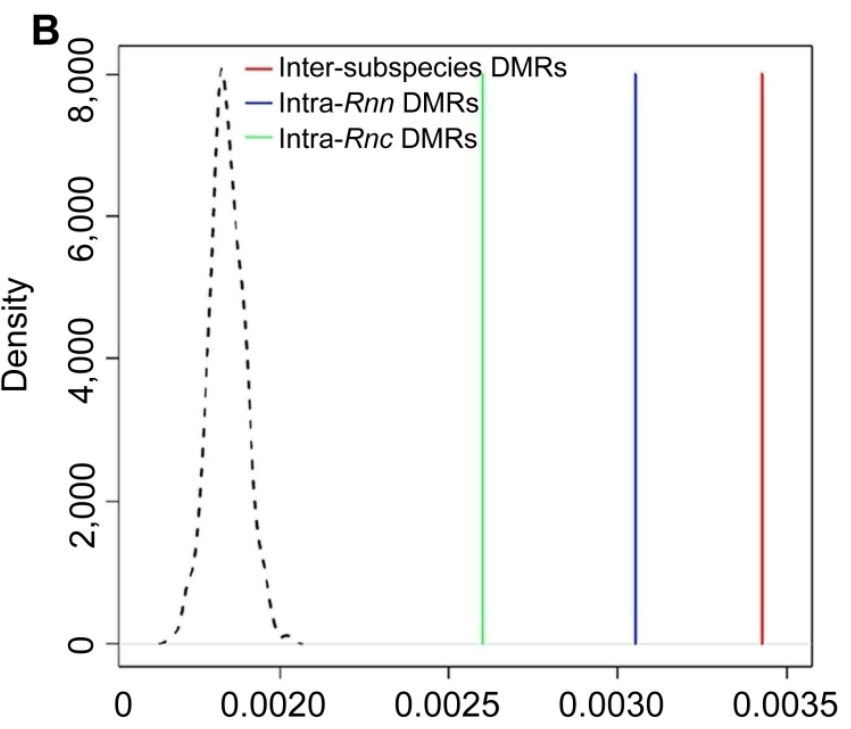

of each subspecies respectively. Coordinates of horizontal axis: 1. Inter-subspecies DMRs; 2. Intra-Rnn DMRs; 3. Intra-Rnc DMRs; 4. Inter-subspecies non-DMRs; 5. Intra-Rnn non-DMRs; 6. Intra-Rnc non-DMRs. Comparisons of $\pi$ in each set are made using a Mann-Whitney test. (B) Comparison of $\pi$ using the first sampling method. 

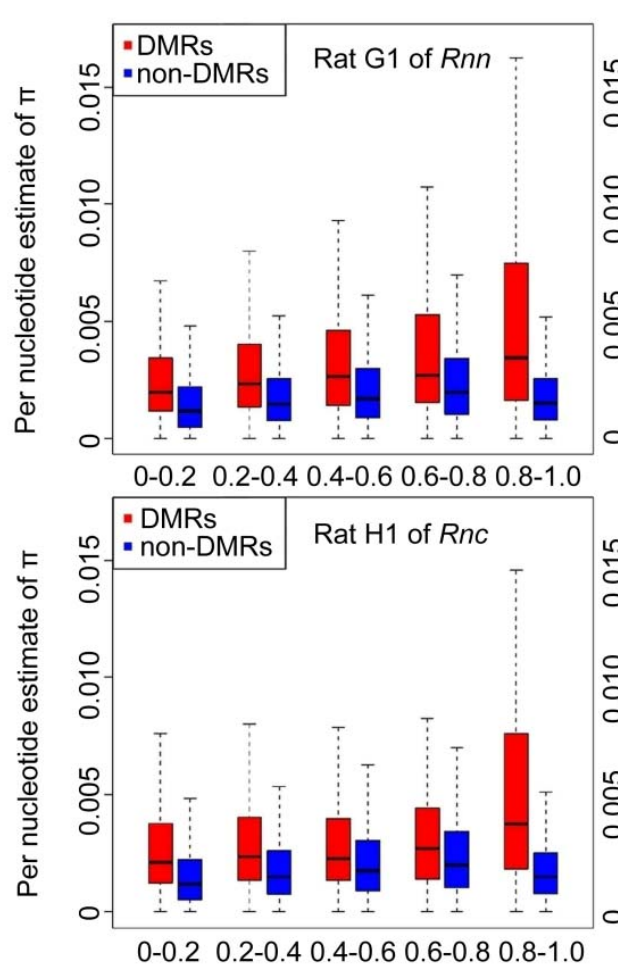

Methlation level
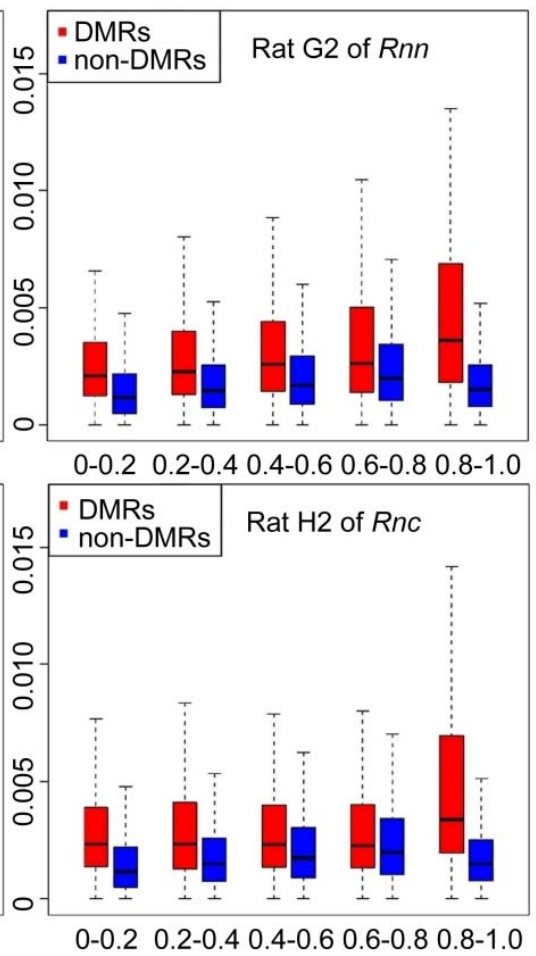

Methlation level
Figure 4. DMRs have a significantly higher $\pi$ than non-DMRs irrespective of the methylation level. Here, $\pi$ is calculated using all 4 individuals. (A/G, A/C, A/T, C/T, C/G and T/G) in inter-subspecies DMRs and non-DMRs, as well as across the whole genome after normalization. The substitution rates of $\mathrm{C} / \mathrm{T}$ and $\mathrm{A} / \mathrm{G}$ were significantly

231 higher than the other four types of DNA substitutions (A/C, T/G, C/G and A/T) both in DMRs

232 and non-DMRs (Independent T test: $p<0.0001$; Fig. 5A) indicating the prevalence of

233 deamination and the higher rate of transitions. However, the normalized rate of each substitution

234 type in inter-subspecies DMRs is 2.5 times higher than that of non-DMRs and of the whole

235 genome (Independent T test: $p<0.0001$; Fig. 5A), suggesting that methylation variation affects

236 all 6 nucleotide substitution types, not just types related to methylation. 
A

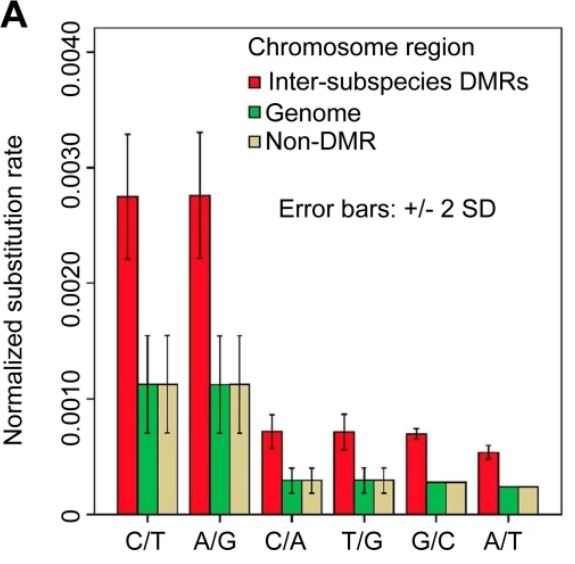

B

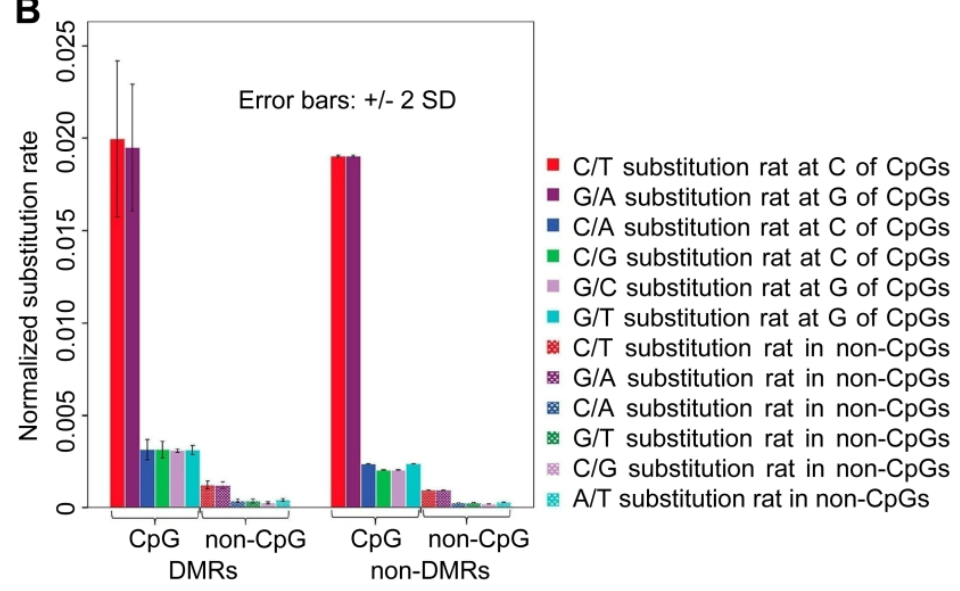

238 Figure 5. Deamination caused by methylation is not the root cause of increased nucleotide

239 diversity in DMRs. (A) Comparison of 6 different types of DNA substitution rates between

240 inter-subspecies DMRs and non-DMRs. (B) Comparison of different types of DNA substitution

241 rate in $\mathrm{CpG}$ and non-CpG sites between inter-subspecies DMRs and non-DMRs using the second

242 sampling method.

Because methylation and deamination mainly occur at the cytosine base in $\mathrm{CpGs}$, the $\mathrm{C} / \mathrm{T}$ substitution rate at these sites, as well as paired G/A on another strand, should be higher than that

246 of non-CpGs. We compared normalized DNA substitution rates in $\mathrm{CpG}$ sites and non-CpG sites

247 between DMRs and non-DMRs (using the second non-DMR sampling method to decrease the

248 influence of sequence length on substitution rate calculation). As expected, the normalized rates

249 of $\mathrm{C} / \mathrm{T}$ and $\mathrm{G} / \mathrm{A}$ substitutions in $\mathrm{CpG}$ are higher than in non-CpGs in both DMRs and non-DMRs

250 (Mann-Whitney test: $p<0.0001$; Fig. 5B), which demonstrates the strong impact of deamination

251 on $\mathrm{C} / \mathrm{T}$ substitutions. We then compared DNA substitution rates in $\mathrm{CpG}$ sites and non-CpG sites

252 between DMRs and non-DMRs, respectively. In non-CpG sites, all 6 normalized DNA

253 substitutions are significantly higher in DMRs than in non-DMRs (Mann-Whitney test: $p<0.01$;

254 Fig. 5B). This result demonstrates that the difference in nucleotide diversity between DMRs and

255 non-DMRs is not a consequence of deamination because non-CpG sites are basically unaffected 
256 by methylation. However, in $\mathrm{CpG}$ sites, neither $\mathrm{C} / \mathrm{T}$ nor $\mathrm{G} / \mathrm{A}$ substitution rates are significantly

257 different between DMRs and non-DMRs (Mann-Whitney test: $p>0.05$; Fig. 5B), whereas the

258 other 4 types of substitution (C/A and $\mathrm{C} / \mathrm{G}$ at cytosine of $\mathrm{CpG} ; \mathrm{G} / \mathrm{T}$ and $\mathrm{G} / \mathrm{C}$ at guanine of $\mathrm{CpG}$ )

259 are significantly higher within DMRs compared to non-DMRs (Mann-Whitney test: $p<0.01$; Fig.

260 5B). This result indicates that methylation-induced deamination cannot account for the increased

261 rates of $\mathrm{C} / \mathrm{T}$ and $\mathrm{G} / \mathrm{A}$ substitutions in DMRs. Meanwhile, the increased rates of $\mathrm{C} / \mathrm{T}$ and $\mathrm{G} / \mathrm{A}$

262 substitutions in DMRs imply that deamination exerts relatively lower influence on $\mathrm{C} / \mathrm{T}$

263 substitution at $\mathrm{CpG}$ sites in DMRs than in non-DMRs. In other words, CpG sites are relatively

264 more conserved in DMRs than in non-DMRs. Thus, the above results support the idea that

265 deamination caused by methylation is not the root cause of increased nucleotide diversity in

266 DMRs, and that it is variance of methylation level but not methylation itself influencing the

267 substitution rates of DMRs.

269 Conserved CpG content in DMRs indicates additional forces drive methylation differences

270 DNA methylation is highly associated with the genomic and functional context

271 (Gutierrez-Arcelus et al. 2013). We characterized CpG contents in DMRs to explore the possible

272 influence of DNA variation on methylation patterns. The average $\mathrm{CpG}$ number per DMR is 27.03

$273 \pm 18.30$ (average density $=0.0248 \pm 0.0180$ ) in inter-subspecies DMRs, $19.01 \pm 7.73$ (average

274 density $=0.0190 \pm 0.0073)$ in intra-Rnn DMRs, $19.95 \pm 7.94$ (average density $=0.0199 \pm 0.0079)$

275 in intra-Rnc DMRs (Supplemental Fig. 5A). The average CpG density of combined DMRs

$276(0.019430 \pm 0.000478)$ is significantly higher than that of non-DMRs $(0.012789 \pm 0.000153)$

277 (Mann-Whitney test: $p<0.00001)$ (Supplemental Fig. 6).

278 The average frequency of variant $\mathrm{CpG}$ sites occurring in a single DMR is $4.15 \%, 4.91 \%$ and

$2792.44 \%$ in inter-subspecies DMRs, intra-Rnn and intra-Rnc DMRs, respectively (Supplemental

280 Table 7). In total, when calculated using the number of variant $\mathrm{CpG}$ sites in each DMR, 70.1\%,

$28185.3 \%$ and $91.5 \%$ of inter-subspecies, intra-Rnn and intra-Rnc DMRs respectively carry no more 
282 than one variant $\mathrm{CpG}$ site and $93.8 \%-99.1 \%$ of the 3 DMR datasets carry no more than three

283 variant $\mathrm{CpG}$ sites (Supplemental Fig. 5B). According to the definition of DMRs and average CpG

284 number of a single DMR, such low variation in CpG sites within DMRs indicates that the DNA

285 methylation level variation is unlikely directly caused by the $\mathrm{CpG}$ variation, but driven by other

286 forces.

\section{Discussion and conclusion}

289 The results from phylogenetic inference, Tajima's D calculations and the ratio of SS-SNVs all

290 indicate strong selective signals within the inter-subspecies DMRs but not intra- subspecies

291 DMRs or non-DMRs. Both inter-subspecies DMRs and intra- subspecies DMRs have higher

292 nucleotide diversity than non-DMRs, however, only inter-subspecies DMRs show accelerated

293 fixation of SNVs. Thus, accelerated DNA evolution in inter-subspecies DMRs is comprised of

294 two independent processes: increased nucleotide diversity and accelerated fixation of these DNA

295 variants.

296 Deamination of methylated cytosines has been suggested as the main mechanism of

297 nucleotide diversity variation across the genome (Fryxell and Moon 2005; Mugal and Ellegren

298 2011; Xia et al. 2012; Zhao and Jiang 2007). Our results demonstrate that increased nucleotide

299 diversity in DMRs is driven by variation of methylation level rather than deamination of

300 methylated cytosines, indicating changed epigenetic status could be the root cause of increased

301 nucleotide diversity in DMRs. A recent review suggested that methylation changes occur

302 downstream of gene regulation during cellular differentiation (Baubec and Schubeler 2014).

303 Similarly, gene expression changes are necessary for methylation changes to occur in response to

304 environmental shifts. This lets us speculate that it is adaptation to the changing environment

305 leading to changes of epigenetic status, which in turn induce the increased nucleotide diversity. A

306 recent study reported that replication defects, which result from chromatin changes caused by a

307 DNMT3B mutation, can cause differences in individuals with mutations (Lana et al. 2012), 
308 indicating a correlation between methylation status and mutation rates. $\mathrm{CpG}$ content dependent

309 correlation between non- $\mathrm{CpG}$ and $\mathrm{CpG}$ mutations (with a threshold of $\sim 0.53 \% \mathrm{CpG}$ content)

310 (Walser et al. 2008; Walser and Furano 2010) also supports a role for methylation in DNA

311 mutation. Epigenetic status may have an impact on the fidelity of DNA replication, causing an

312 increased replication error rate (Loeb and Monnat 2008; McCulloch and Kunkel 2008; Walser et

313 al. 2008; Walser and Furano 2010). This implies that replication could be affected by chromatin

314 changes. Germ line DNA mutations occur mainly during replication in meiosis. As reviewed in

315 previous studies, alteration of the sperm epigenome, including DNA methylation, histone

316 modification and sRNA have now been shown to be a mechanism of transgenerational

317 inheritance (Boffelli and Martin 2012; Heard and Martienssen 2014; Lim and Brunet 2013), and,

318 these changes do affect chromatin status. Additionally, reprogramming finishes during meiosis

319 (Seisenberger et al. 2013), and methylation status is faithfully maintained during DNA replication

320 in meiosis. Thus, these data support a model in which adaptation to the changing environment

321 leads to changes in epigenetic status, which in turn induces higher nucleotide diversity (Fig. 6). 


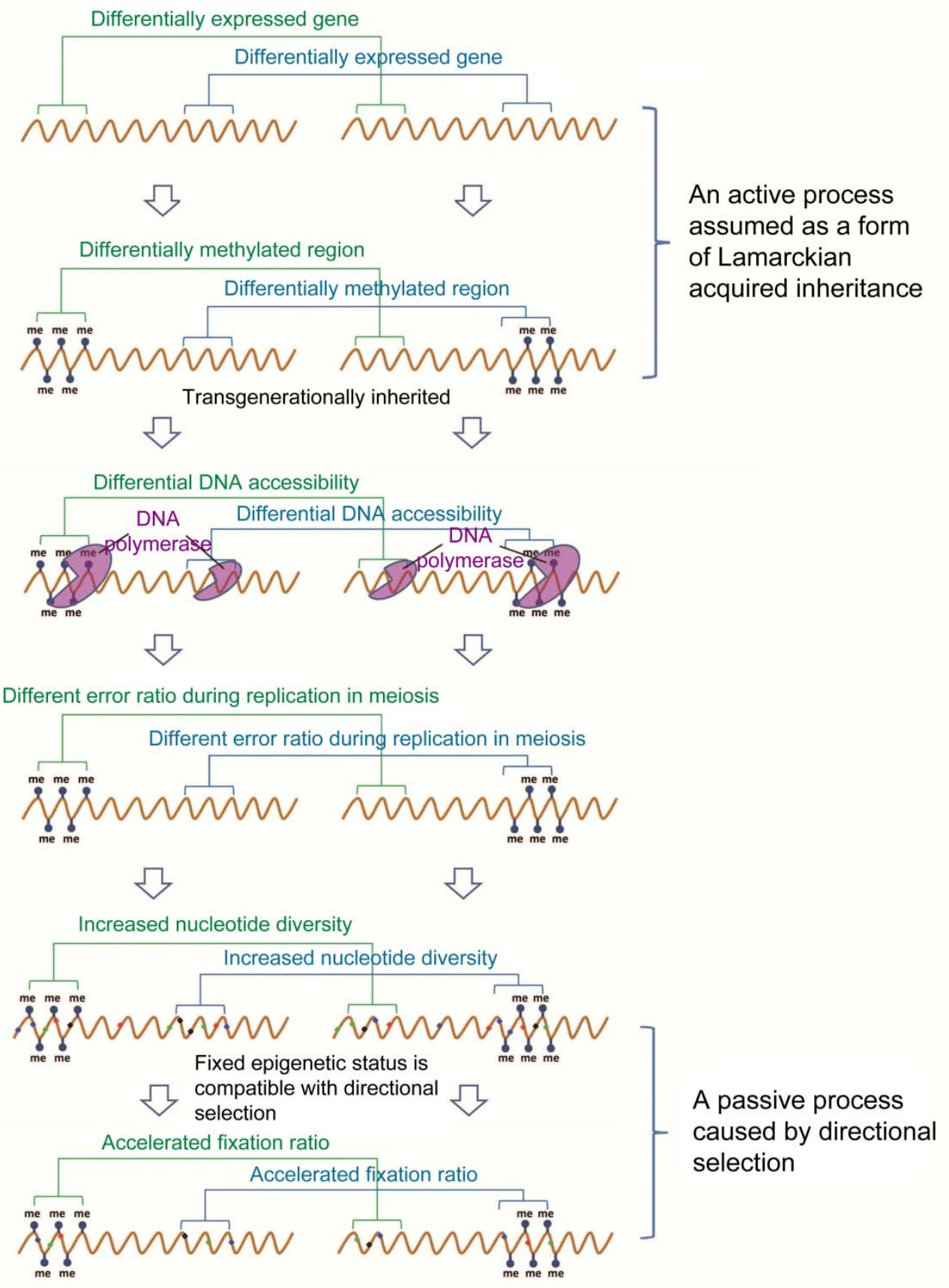

323 Figure 6.Hypothesized model of increased nucleotide diversity and accelerated fixation ratio in

324 DMRs. Schematic of the model in which environmentally induced methylation differences lead to 
325 increased DNA substitutions and accelerated fixation. Orange lines represent DNA, and colored 326 dots on the DNA represent substitutions.

327 An alternative hypothesis is that the DNA sequence in DMRs may be mutation hotspots,

328 which may drive compatible variation of methylation status as suggested previously (Liu et al.

329 2014). However, three key pieces of evidence make this unlikely. First, we found significantly

330 increased nucleotide diversity between subspecies but decreased nucleotide diversity within each

331 subspecies in inter-subspecies DMRs. Second, in intra-Rnn DMRs, comparatively the majority of

332 these regions are non-DMRs in Rnc (Supplemental Fig. 1), we only see increased nucleotide

333 diversity between the two individuals of subspecies Rnn but not Rnc, and vice versa. Third,

334 sampled non-DMRs with similar nucleotide composition but low $\mathrm{CpG}$ density indicate the strong

335 relationship between increased nucleotide diversity and $\mathrm{CpG}$ density. However, $\mathrm{CpG}$ content is

336 relatively more conserved in DMRs than in non-DMRs. Thus, we can conclude that it is variation

337 of methylation level inducing changes of chromatin accessibility, which in turn leads to increased

338 nucleotide diversity. Changing the underlying DNA sequence can be a slow process that isn't

339 dynamic enough to respond to rapid environmental change (Heard and Martienssen 2014),

340 especially for complex traits. Logically, methylation changes caused by active regulation of gene

341 expression in response to changing environment should occur prior to increased DNA variation,

342 because DNA mutation is a random process that can't support dynamic gene expression

343 regulation.

344 Directional selection increases the fixation probability of DNA variants. However,

345 accelerated fixation of DNA variants occurs only in inter-subspecies DMRs. Since both

346 intra-subspecies DMRs (caused by random and temporary environmental changes) and

347 inter-subspecies DMRs (caused by stable and long-standing environmental changes) show

348 increased nucleotide diversity, this is unlikely to be a consequence of natural selection. However,

349 increased nucleotide diversity may provide a substrate for natural selection to act upon. 
350 The subspecies-specific methylation status in inter-subspecies DMRs implies that

351 methylation statuses can be maintained by stable environment differences. Additionally, because

352 we saw accelerated fixation in inter-subspecies DMRs but not intra-subspecies DMRs with

353 similar nucleotide diversity, we speculated that stably diverged environments act as a kind of

354 directional selection, leading to fixation of specific gene expression patterns and associated

355 epigenetic status, which eventually fix advantageous DNA variants (Fig. 6).

356 Epigenetic inheritance can be compatible with Darwinian evolution if epigenetic statuses

357 that specify traits can be transgenerationally inherited (Boffelli and Martin 2012). Our results

358 demonstrate that not only environment-associated methylation variation can be maintained and

359 transgenerationally inherited, but also lead to increasing of nucleotide diversity. We show that

360 fixed methylation status is compatible with Darwinian evolution, and can lead to accelerated

361 fixation of DNA variants that confer an advantage in the new environment. These results

362 establish a bridge between Lamarckian acquired inheritance and Darwinian selection. 


\section{Experimental Paradigm}

367 The two selected subspecies, $R$. n. caraco and $R$. n. norvegicus, live in extremely different

368 environments from north and south China, respectively. Harbin City has a severe winter

$369\left(126^{\circ} 32^{\prime} \mathrm{E}, 45^{\circ} 48^{\prime} \mathrm{N}\right)$ compared to Zhanjiang City $\left(110^{\circ} 21^{\prime} \mathrm{E}, 21^{\circ} 16^{\prime} \mathrm{N}\right)$, which is the main reason

370 for reproduction inhibition in the subspecies $R$. n. caraco. Two individuals were selected for each

371 subspecies, and both the genome and the sperm methylome were sequenced for each sample. We

372 proofread the methylomes using the genome sequence of each individual to ensure the statistical

373 reliability of relationship between methylation and DNA variation.

374 We examined DMRs with methylation level changes greater than two fold and methylation

375 level differences (MLD) greater than or equal to 0.2 in the methylomes between the two

376 subspecies (inter-subspecies DMRs), or within R. n. caraco (intra-Rnc DMRs) and R. $n$.

377 norvegicus (intra-Rnn DMRs), respectively. Inter-subspecies DMRs are defined as DNA regions

378 with significantly higher inter-subspecies MLD than intra-subspecies MLD (see Differential

379 Methylation Analysis). The genomic regions excluding the inter- and intra-subspecies DMRs are

380 defined as non-DMRs. Genome sequences were classified as DMRs and non-DMRs for further

381 analysis.

382 Because nucleotide diversity $(\pi)$ and other indices of variation are sensitive to the length of

383 DNA sequences, two methods were used for sampling of non-DMRs as follows: First, non-DMRs

384 with equal length and GC content to each unique inter-subspecies DMR set are randomly sampled,

385 and then the sampled 1859 non-DMRs are combined into one sequence, with length equal to the

386 total length of inter-subspecies DMRs $(2.6 \mathrm{Mb})$. This sampling process is repeated 1,000 times,

387 and decreases the influence of sequence length on $\pi$ and other statistics. Second, because the

388 mean length of DMRs is less than $1.5 \mathrm{~kb}$, non-DMRs with equal length and GC content to each

389 unique DMR are randomly sampled 10,000 times from the genome, and the mean $\pi$ of these

390 sampled non-DMRs is used as the value of the non-DMR corresponding to that unique DMR to 
391 reduce the randomness and bias of sampled non-DMRs. This method enables comparison of the

392 landscape of variation in a single DMR within the three DMR datasets.

\section{Sperm Collection and DNA extraction}

395 All animal experiments were conducted with the permission of the Institutional Animal Use and 396 Care Committee of the Institute of Plant Protection, Chinese Academy of Agricultural Sciences.

397 Mature sperm were isolated from cauda epididymides as described by Kempinas with some 398 modifications (Kempinas and Lamano-Carvalho 1988). The cauda epididymidis was cut 399 longitudinally in a 35mm diameter Petri dish with 3ml M2 medium (M7167, Sigma). The sperm 400 were released by repeatedly and gently disrupting using a pipette tip after incubation at room 401 temperature for 20min, and were then collected and washed twice in PBS. To eliminate somatic 402 cell contamination, the sperm were treated with somatic cell lysis buffer $(0.1 \%$ SDS, $0.5 \%$ Triton $403 \mathrm{X}$ in DEPC H2O) for 20 min on ice (Peng et al. 2012). Purified sperm were then incubated in 404 SNET buffer (20 mM Tris-HCl, 5 mM EDTA, 400 mM NaCl, 1\% (wt/vol) SDS; pH 8.0) 405 containing $400 \mu \mathrm{g} / \mathrm{ml}$ Proteinase $\mathrm{K}$ and $40 \mathrm{mM}$ DTT in a $55^{\circ} \mathrm{C}$ shaker $(\approx 150)$ overnight. DNA was 406 harvested using a standard phenol-chloroform extraction protocol. For extraction of DNA from 407 testis, the SNET buffer with only Proteinase K was used, followed by the standard extraction 408 protocol. DNA was eluted in TE for sequencing.

\section{DNA Isolation, BS-Seq Library Construction and Sequencing}

411 Five $\mu \mathrm{g}$ genomic DNA was first fragmented by sonication with a Covaris S2 system (Covaris,

412 MA) to a mean size of approximately $250 \mathrm{bp}$, followed by end repair, 3'-end addition of dA, and 413 adapter ligation. Methylated adapters were used according to the manufacturer's instructions

414 (Illumina). The bisulfite conversion of sample DNA was carried out using a modified

415 NH4HSO3-based protocol 1 and amplified with 9 cycles of PCR. Paired-end sequencing was 416 carried out using an Illumina HiSeq 2000. 


\section{Genome re-sequencing and BS-Seq Analysis}

419 The Rattus norvegicus reference genome was downloaded from Ensemble (release-70). To

420 avoid the failure of reads mapping caused by additional mismatches resulting from $\mathrm{C}$ to $\mathrm{T}$

421 transitions after bisulfite treatment, all Cs in the reference genome were converted to Ts (T-genome)

422 and all Gs were converted to As (A-genome) separately, creating two reference genomes.

423 Moreover, the sequenced reads were prepared for alignment by replacing observed Cs on the

424 forward read with Ts and observed Gs on the reverse reads with As. We used SOAP2 (Version 2.21)

425 (Li et al. 2008; $\mathrm{Li}$ et al. 2009) to map the transformed reads to both the $\mathrm{T}$ - and $\mathrm{A}$ - genomes,

426 allowing up to 6 mismatches in 90 bp paired-end reads. Reads aligning to more than one position on

427 the genome were discarded. Multiple reads mapping to the same position were regarded as PCR

428 duplicates, and only one of them was kept. For $\mathrm{mC}$ detection, we retrieved the original sequence of

429 the transformed reads and compared it with the untrasformed reference genomes. Cytosines in

430 BS-seq reads that matched to $\mathrm{Cs}$ on the reference were counted as potential mCs. Cytosines with a

431 quality score $<20$ were not considered.

432 The bilsulfite conversion rate of each library was calculated as the total number of sequenced

433 Cs divided by the total sequencing depth for sites corresponding to Cs in non-CpG sites. The

434 conversion rates of all the libraries was higher than $99 \%$. To distinguish true positives from false

435 positives, we used a model based on the binomial distribution $\mathrm{B}(\mathrm{n}, \mathrm{p})$, with $\mathrm{p}$ equal to the false

436 positive rate and $\mathrm{n}$ equal to the coverage depth of each potential $\mathrm{mC}$. For example, given a potential

$437 \mathrm{mC}$ position with $\mathrm{k}$ sequenced cytosines and total depth of $\mathrm{n}$, we calculated the probability that all

438 the $\mathrm{k}$ cytosines sequenced out of $\mathrm{n}$ trials were false positives, and then compared the probability of

$439 \mathrm{~B}(\mathrm{k}, \mathrm{n}, \mathrm{p})$ to 0.01 after adjusting $p$-values by the FDR method (Benjamini et al. 2001). Only the mCs

440 with adjusted $p$-values $<0.01$ were considered true positives. 
444 The methylation level of an individual cytosine was determined by the number of reads containing

$445 \mathrm{a} \mathrm{C}$ at the site of interest divided by the total number of reads containing the site. Methylation level

446 of a specific region was determined by the sum of methylation levels of individual cytosines in the

447 region divided by the total number of covered cytosines in this region.

\section{Differential Methylation Analysis}

450 Two-way analysis of variance (two-way ANOVA) was conducted to identify differentially 451 methylated regions (DMRs) between two groups of samples (i.e. G1+G2 vs H1+H2) using 200, $452500,800,1,000,2,000,3,000,4,000,5,000,10,000$ bp sliding windows with step lengths of $50 \%$ of

453 the window size. As methylation at $\mathrm{CpG}$ sites is symmetric, we combined the data from the plus 454 and minus strands for each CpG site during DMR detection. To ensure adequate power in the 455 statistical test, only windows with at least 6 informative CpGs ( $\geq 5 \mathrm{X}$ coverage) in all four 456 sequenced samples were considered. The two independent variables for ANOVA were group and 457 cytosine position. For each window, we first calculated the variance between groups (variance 458 caused by inter-group differences) and the variance between two individuals within the same group 459 (variance caused by inter-individual differences), then used an F-test to calculate the $p$-value of 460 each window by comparing the inter-group variance and inter-individual variance. $P$-values were 461 then adjusted for multiple testing by the FDR method (Benjamini et al. 2001). Only windows with 462 adjusted $p$-value $<0.05$ and $>2$-fold methylation level change were considered as candidate DMRs.

463 In addition, we removed all DMRs in which the differences in methylation levels were $<0.2$.

464 Finally, contiguous DMRs and DMRs identified with different window sizes were merged.

466 Normalization Procedures for Rates of Each Substitution Type

467 The substitution rates of any SNV site was normalized as follows:

$468 \quad \mathrm{C} / \mathrm{T}$ site as example: 


$$
S R_{c / t}=\left(\frac{c t_{n c}}{c_{n}}+\frac{t c_{n c}}{t_{n}}+\frac{c t_{c n}}{c_{c}}+\frac{t c_{c n}}{t_{c}}\right) / 4
$$

471 substitutions when comparing the genome of Rnn to genome of Rnc. The ${ }^{c_{n}}$ is the total number of

472 cytosines in the two Rnn genomes. The $t c_{n c}$ is the total number of $\mathrm{T}$ to $\mathrm{C}$ substitutions when

473 comparing the genome of Rnn to genome of Rnc. The ${ }^{t}$ is the total number of thymines in the two

474 Rnn genomes. The $c t_{c n}$ is the total number of $\mathrm{C}$ to $\mathrm{T}$ substitution reading from genome of Rnc to

475 genome of Rnn. The $c_{c}$ is the total number of cytosines in the two Rnc genomes. The $t c_{c n}$ is the

476 total number of T to C substitutions when comparing the genome of Rnc to genome of Rnn. The $t_{c}$

477 is the total number of thymines in the two Rnc genomes. Others and so on.

478 Substitution rates in $\mathrm{CpG}$ were normalized using the combined length of $\mathrm{CpGs}$, and 479 substitution rates of non-CpG were normalized using the length of non-CpG nucleotides.

\section{$481 \quad$ Population test of RSS}

482 RSS (ratio of SS-SNVs) is the percentage of subspecies specific SNVs (SS-SNVs, Supplemental

483 Fig. 3) among all SNVs. SS-SNVs was defined as sites with allele frequency from $0.25-0.5$

484 (calculated by 8 chromosomes) and detected only in one of two subspecies but both two

485 individuals. To increase the accuracy of RSS calculation, we added 10 genome sequences (5 for

486 each subspecies, kindly provided by the Kunming Institute of Zoology, CAS), with about 3x

487 coverage for further analysis. In the early study, we identified a total of $12,669,132$ SNVs in the 4

488 genomes, among which, 3,236,117 (25.54\%) are covered in the 14 genome data. For the 20,634

489 SNVs in inter-subspecies DMRs, 4300 (20.84\%) are covered in the 14 genome data (Table 1). 
We tested the allele distribution of each SNV site covered in the 14 genomes using the

491 Chi-squared test with Yates' continuity correction, and sites with $p<0.05$ are defined as

492 SS-SNVs. We further compared the difference of RSS between inter-subspecies DMRs and

493 non-DMRs using the Chi-squared test with Yates' continuity correction.

\section{Computational Methods}

496 All DNA phylogenetic trees were constructed using the Neighbor-Joining method in the PHYLIP

497 software package. Substitution model: Jukes-Cantor, Rates: uniform rates. We calculated

498 nucleotide diversity using method described by Vernot et al (Vernot et al. 2012). Tajima's D was

499 calculated using program in software Mega-CC 6.0 (Kumar et al. 2012). CpG density was

500 calculated as the number of $\mathrm{CpGs} / \mathrm{DMR}$ length. Statistical analyses were performed using R

501 package. The means plus or minus one standard deviation are reported unless otherwise noted.

503 Data access

504 Data analyzed herein have been deposited in SRA with accession XXXXXXXX.

\section{Acknowledgements}

507 We thank Joshua M. Akey from Department of Genome Sciences, University of Washington,

508 USA for advice on genomic analysis strategies and explanation of results. We thank Rachel

509 Gittelman and Wenqing Fu from the Department of Genome Sciences, University of Washington,

510 USA, and Guoliang Wang from Institute of Plant Protection, Chinese Academy of Agricultural

511 Sciences, China, for advice on manuscript preparation. We thank Dongdong Wu and Lin Zeng

512 from Kunming Institute of Zoology, Chinese Academy of Science, China for providing additional

51310 genome sequence data used for population test. This experiment was supported by The

514 Agricultural Science and Technology Innovation Program, National Key Technology R\&D 
515 Program (2012BAD19B02), and National Basic Research Program of China (973 Program,

516 2007CB109104).

517

\section{Author Contributions}

519 XH.L. designed all experiments. XH.L., F.L., N.L., DW.W., and Y.S. prepared the figures and

520 wrote the manuscript. XH.L., JM.L., QY.L., YB.J., and Y.S. performed genomic and statistical

521 analysis. F.L., DW.W., N.L., and QY.L. contributed to design of the experiment. ZY.F., and L.C.

522 contributed to the design of the project. DW.W., N.L., DD.Y., JJ.S., and L.C. performed sampling,

523 sperm collection and other preparation of genome sequencing and BS-seq.

524

\section{Disclosure declaration}

526 The funders had no role in study design, data collection, and analysis, decision to publish, or

527 preparation of the manuscript. The authors declare no competing financial interests.

528

529 


\section{References}

531 Baubec T, Schubeler D. 2014. Genomic patterns and context specific interpretation of DNA 532 methylation. Curr Opin Genet Dev 25: 85-92.

533 Benjamini Y, Drai D, Elmer G, Kafkafi N, Golani I. 2001. Controlling the false discovery rate in 534 behavior genetics research. Behav Brain Res 125: 279-284.

535 Boffelli D, Martin DI. 2012. Epigenetic inheritance: a contributor to species differentiation? DNA $536 \quad$ Cell Biol 31 Suppl 1: S11-S16.

537 Bonasio R, Li Q, Lian J, Mutti NS, Jin L, Zhao H, Zhang P, Wen P, Xiang H, Ding Y et al. 2012. 538 Genome-wide and caste-specific DNA methylomes of the ants Camponotus floridanus and Harpegnathos saltator. Curr Biol 22: 1755-1764.

540 Daxinger L, Whitelaw E. 2012. Understanding transgenerational epigenetic inheritance via the 541 gametes in mammals. Nat Rev Genet 13: 153-162.

542 Ehrlich M, Norris KF, Wang RY, Kuo KC, Gehrke CW. 1986. DNA cytosine methylation and 543 heat-induced deamination. Biosci Rep 6: 387-393.

544 Franklin TB, Mansuy IM. 2010. Epigenetic inheritance in mammals: evidence for the impact of 545 adverse environmental effects. Neurobiol Dis 39: 61-65.

546 Fryxell KJ, Moon WJ. 2005. CpG mutation rates in the human genome are highly dependent on $547 \quad$ local GC content. Mol Biol Evol 22: 650-658.

548 Gutierrez-Arcelus M, Lappalainen T, Montgomery SB, Buil A, Ongen H, Yurovsky A, Bryois J, 549 Giger T, Romano L, Planchon A et al. 2013. Passive and active DNA methylation and the 550 interplay with genetic variation in gene regulation. Elife 2: e523.

551 Heard E, Martienssen RA. 2014. Transgenerational epigenetic inheritance: myths and 552 mechanisms. Cell 157: 95-109.

553 Jiang C, Han L, Su B, Li WH, Zhao Z. 2007. Features and trend of loss of promoter-associated $554 \quad$ CpG islands in the human and mouse genomes. Mol Biol Evol 24: 1991-2000. 
555 Kempinas WG, Lamano-Carvalho TL. 1988. A method for estimating the concentration of 556 spermatozoa in the rat cauda epididymidis. Lab Anim 22: 154-156.

557 Kumar S, Stecher G, Peterson D, Tamura K. 2012. MEGA-CC: computing core of molecular 558 evolutionary genetics analysis program for automated and iterative data analysis. 559 Bioinformatics 28: 2685-2686.

560 Lana E, Megarbane A, Tourriere H, Sarda P, Lefranc G, Claustres M, De Sario A. 2012. DNA 561 replication is altered in Immunodeficiency Centromeric instability Facial anomalies (ICF) 562 cells carrying DNMT3B mutations. Eur J Hum Genet 20: 1044-1050.

563 Li R, Li Y, Kristiansen K, Wang J. 2008. SOAP: short oligonucleotide alignment program. $564 \quad$ Bioinformatics 24: 713-714.

565 Li R, Yu C, Li Y, Lam TW, Yiu SM, Kristiansen K, Wang J. 2009. SOAP2: an improved 566 ultrafast tool for short read alignment. Bioinformatics 25: 1966-1967.

567 Lim JP, Brunet A. 2013. Bridging the transgenerational gap with epigenetic memory. Trends $568 \quad$ Genet 29: 176-186.

569 Liu Y, Li X, Aryee MJ, Ekstrom TJ, Padyukov L, Klareskog L, Vandiver A, Moore AZ, Tanaka 570 T, Ferrucci L et al. 2014. GeMes, clusters of DNA methylation under genetic control, can 571 inform genetic and epigenetic analysis of disease. Am J Hum Genet 94: 485-495.

572 Loeb LA, Monnat RJ. 2008. DNA polymerases and human disease. Nat Rev Genet 9: 594-604.

573 McCulloch SD, Kunkel TA. 2008. The fidelity of DNA synthesis by eukaryotic replicative and $574 \quad$ translesion synthesis polymerases. Cell Res 18: 148-161.

575 Mugal CF, Ellegren H. 2011. Substitution rate variation at human CpG sites correlates with 576 non-CpG divergence, methylation level and GC content. Genome Biol 12: R58.

577 Peng H, Shi J, Zhang Y, Zhang H, Liao S, Li W, Lei L, Han C, Ning L, Cao Y et al. 2012. A 578 novel class of tRNA-derived small RNAs extremely enriched in mature mouse sperm. Cell $579 \quad \operatorname{Res} 22: 1609-1612$. 
580 Seisenberger S, Peat JR, Hore TA, Santos F, Dean W, Reik W. 2013. Reprogramming DNA

581 methylation in the mammalian life cycle: building and breaking epigenetic barriers. Philos

$582 \quad$ Trans R Soc Lond B Biol Sci 368: 20110330.

583 Shao C, Li Q, Chen S, Zhang P, Lian J, Hu Q, Sun B, Jin L, Liu S, Wang Z et al. 2014.

584 Epigenetic modification and inheritance in sexual reversal of fish. Genome Res 24: 604-615.

585 Song Y, Lan Z, Kohn MH. 2014. Mitochondrial DNA phylogeography of the Norway rat. PLoS

$586 \quad$ One 9: e88425.

587 Van Soom A, Peelman L, Holt WV, Fazeli A. 2014. An introduction to epigenetics as the link 588 between genotype and environment: a personal view. Reprod Domest Anim 49 Suppl 3: 2-10.

589 Vernot B, Stergachis AB, Maurano MT, Vierstra J, Neph S, Thurman RE, Stamatoyannopoulos

590 JA, Akey JM. 2012. Personal and population genomics of human regulatory variation.

$591 \quad$ Genome Res 22: 1689-1697.

592 Walser JC, Ponger L, Furano AV. 2008. CpG dinucleotides and the mutation rate of non-CpG DNA. Genome Res 18: 1403-1414.

594 Walser JC, Furano AV. 2010. The mutational spectrum of non-CpG DNA varies with CpG $595 \quad$ content. Genome Res 20: 875-882.

596 Wang D, Cong L, Yue L, Huang B, Zhang J, Wang Y, Li N, Liu X. 2011. Seasonal variation in 597 population characteristics and management implications for brown rats (\&lt;i\&gt;Rattus 598 norvegicus\&lt;/i\&gt;) within their native range in Harbin, China. Journal of Pest Science 84: $599 \quad 409-418$.

600 Wu X, Wang Y. 2012. Fossil materials and migrations of Mus musculus and Rattus norvegicus. $601 \quad$ Research of China's Frontier Archaeology 1: 343-353.

602 Xia J, Han L, Zhao Z. 2012. Investigating the relationship of DNA methylation with mutation 603 rate and allele frequency in the human genome. BMC Genomics 13 Suppl 8: S7.

604 Zhao Z, Jiang C. 2007. Methylation-dependent transition rates are dependent on local sequence 605 lengths and genomic regions. Mol Biol Evol 24: 23-25. 


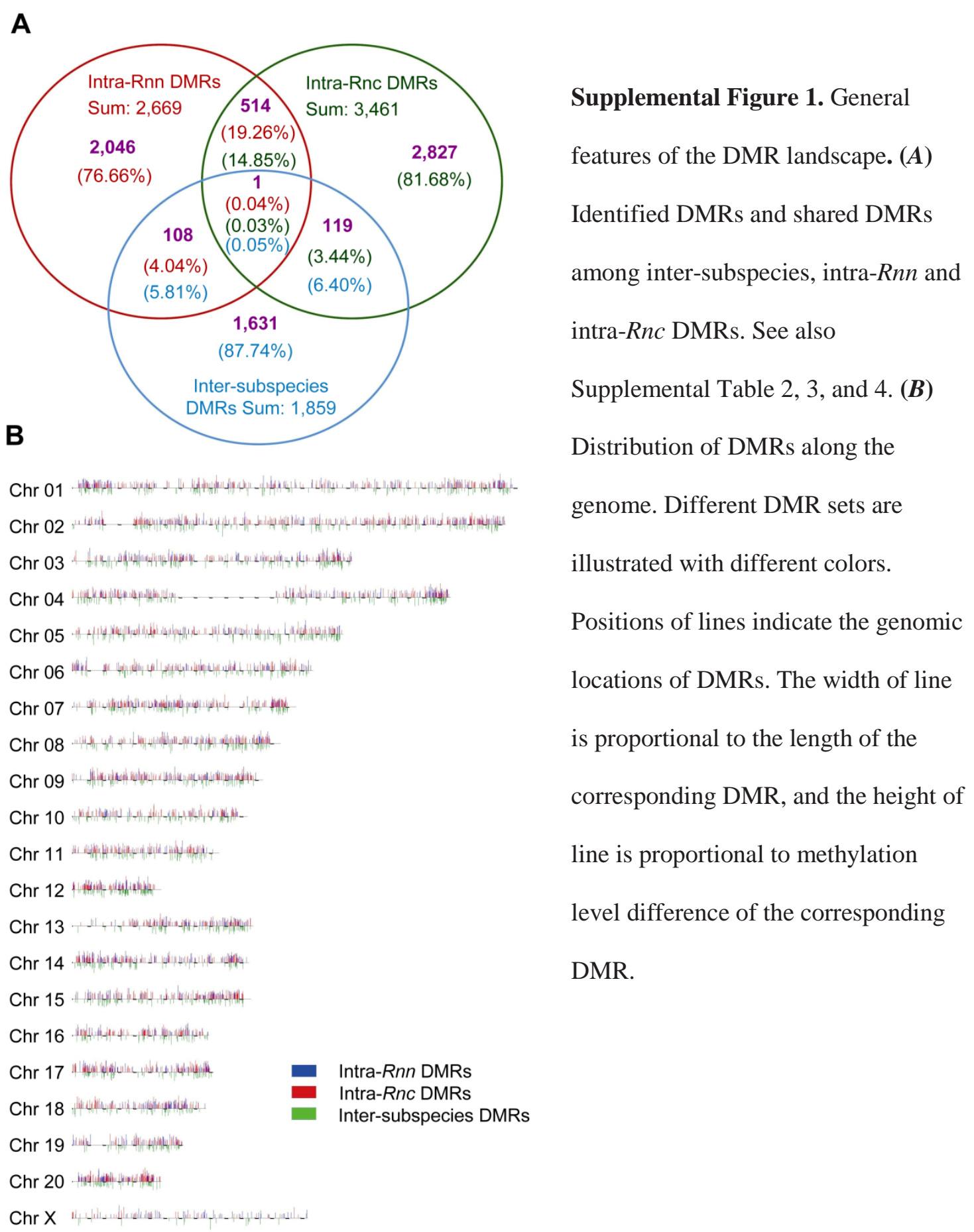




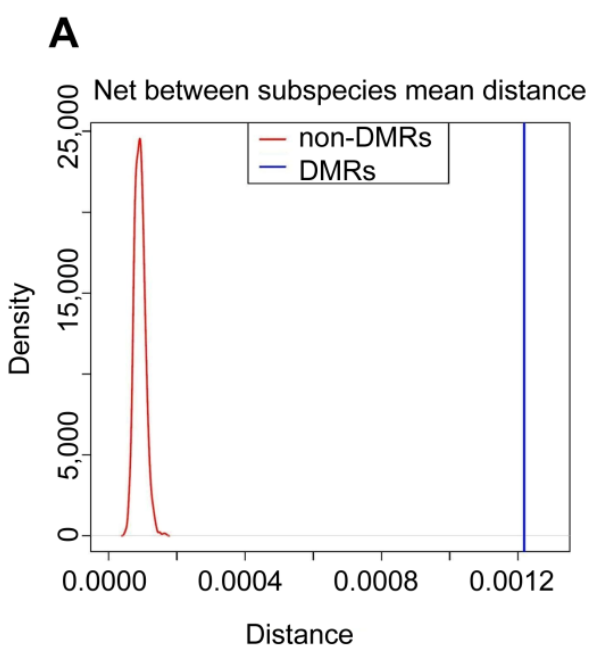

B

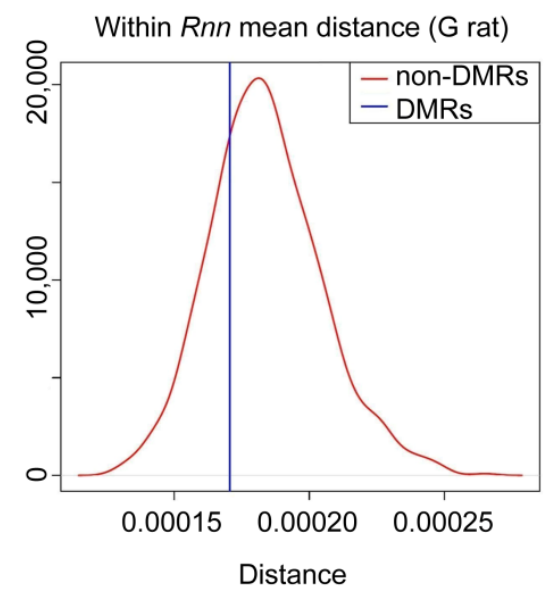

C

Within Rnc mean distance (H rat)

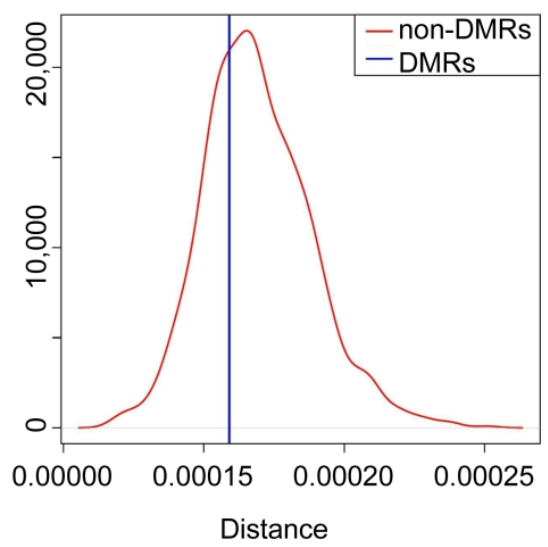

Supplemental Figure 2 Comparisons of between and within group distances between intersubspecies DMRs and non-DMRs, Related to Fig. 1. (A) Comparison of between subspecies distance in inter-subspecies DMRs and non-DMRs. (B) Comparison of within Rnn distance (G1 vs G2) in inter-subspecies DMRs and non-DMRs. (C) Comparison of within Rnc distance (H1 vs H2) in inter-subspecies DMRs and non-DMRs. 


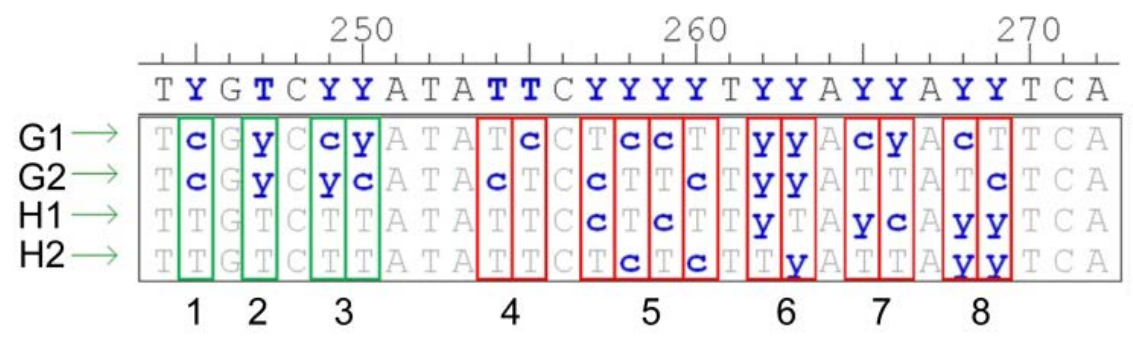

Supplemental Figure 3. Illustration of subspecies specific SNVs, Related to Supplemental Experimental procedures. Because only two individuals of each subspecies were used to genome sequencing, we define SS-SNVs as sites with an allele frequency of $0.25-0.5$ (calculated with 8 chromosomes) and detected in both individuals of one subspecies, but in neither individual of the second subspecies. Thus, SS-SNVs are sites as follows: Site1: The subspecies are fixed for two different alleles. Site 2: Both individuals are heterozygous in one subspecies, while in the other subspecies one allele is fixed. Site 3: One subspecies is fixed for an allele, while in the other subspecies one individual is homozygous for the other allele, and the other individual is heterozygous. In sites 4,5,6 and 7, there aren't any variants that are private to one subspecies and present in both individuals, and they all are not SS-SNVs. 


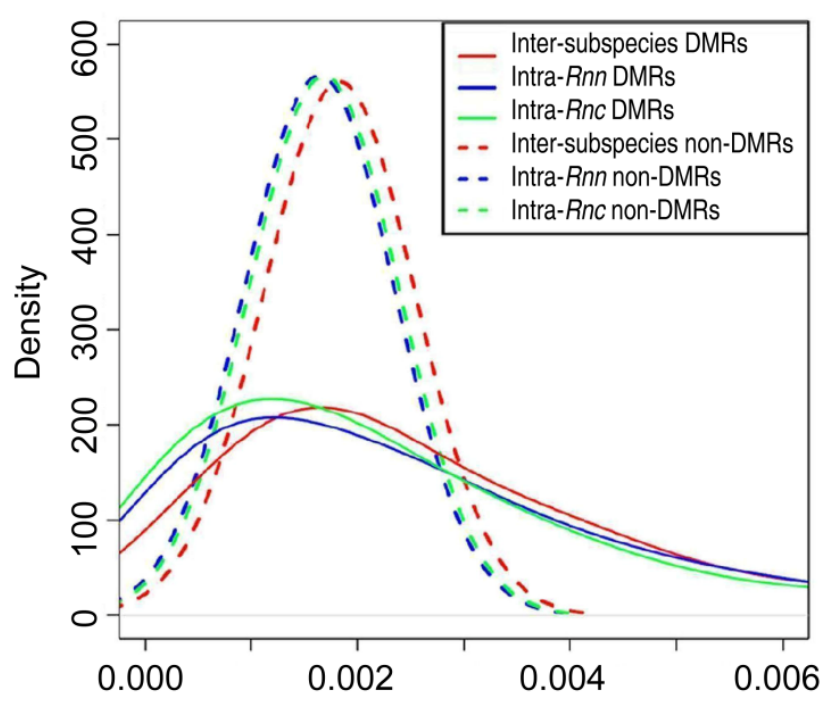

Per nucleotide estimate of $\pi$

Supplemental Figure 4. Distribution of $\pi$ using the second sampling method. $\pi$ of intersubspecies DMRs are calculated using 4 individuals, and $\pi$ of intra-subspecies DMRs are calculated using 2 individuals of each subspecies respectively. 
A
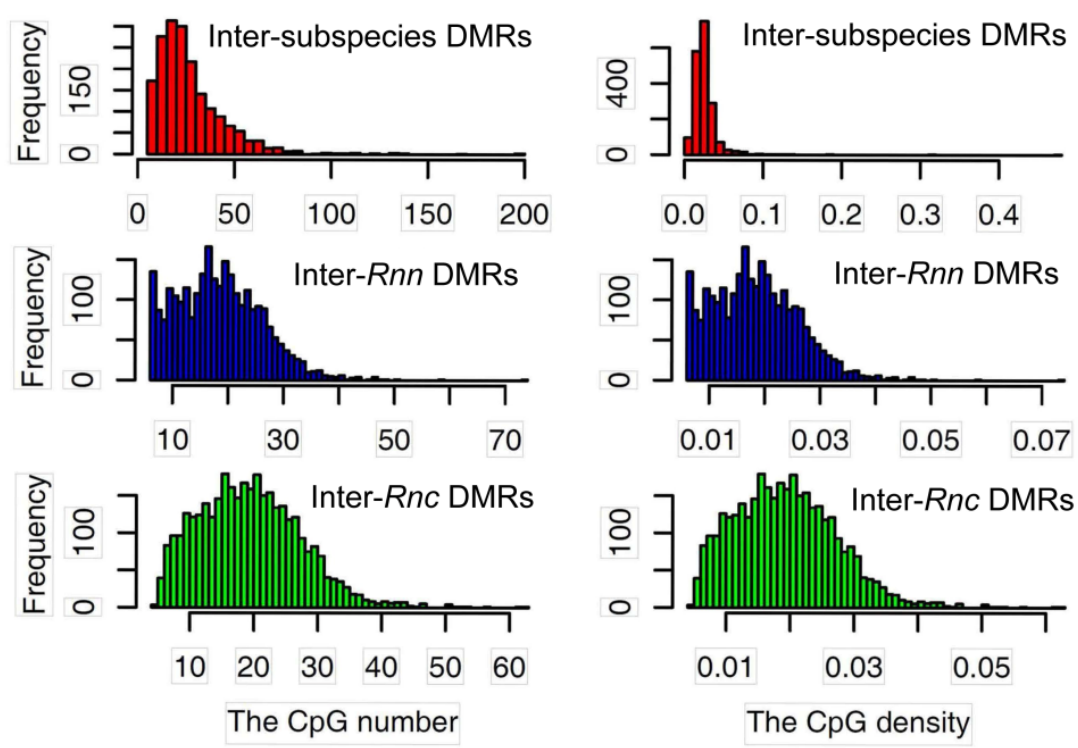

B
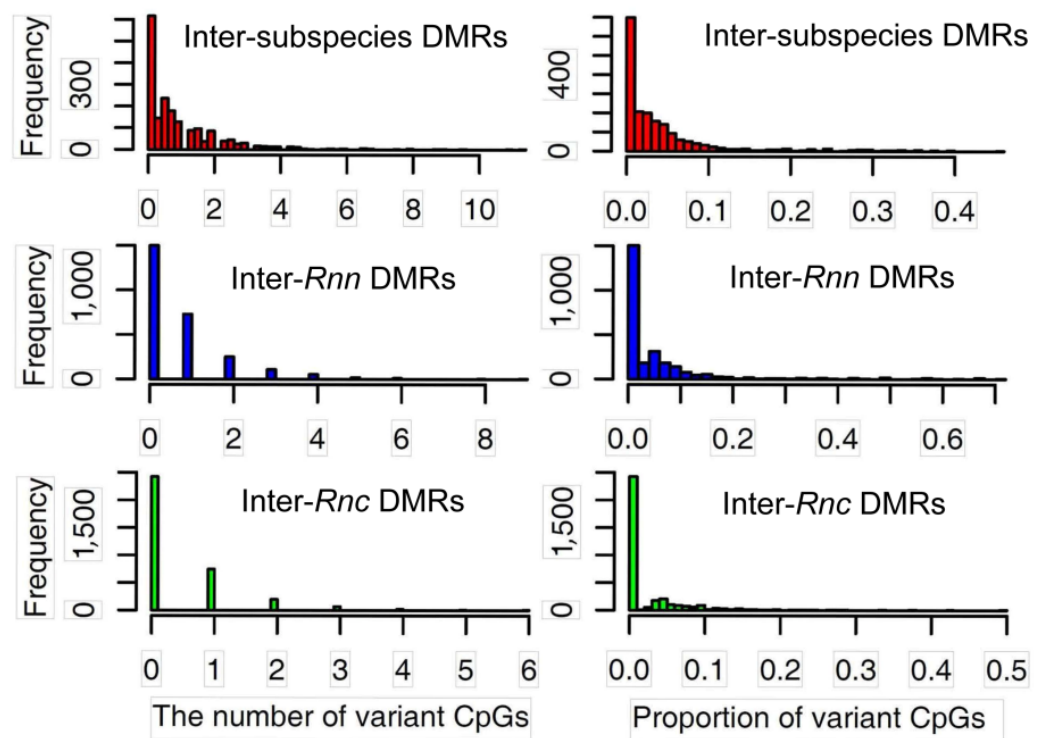

Supplemental figure 5. Characteristics of CpG content of DMRs. (A) Distribution of CpG numbers and densities within inter-subspecies DMRs, intra-Rnn DMRs and intra-Rnc DMRs. (B) Variation of CpGs in DMRs. 


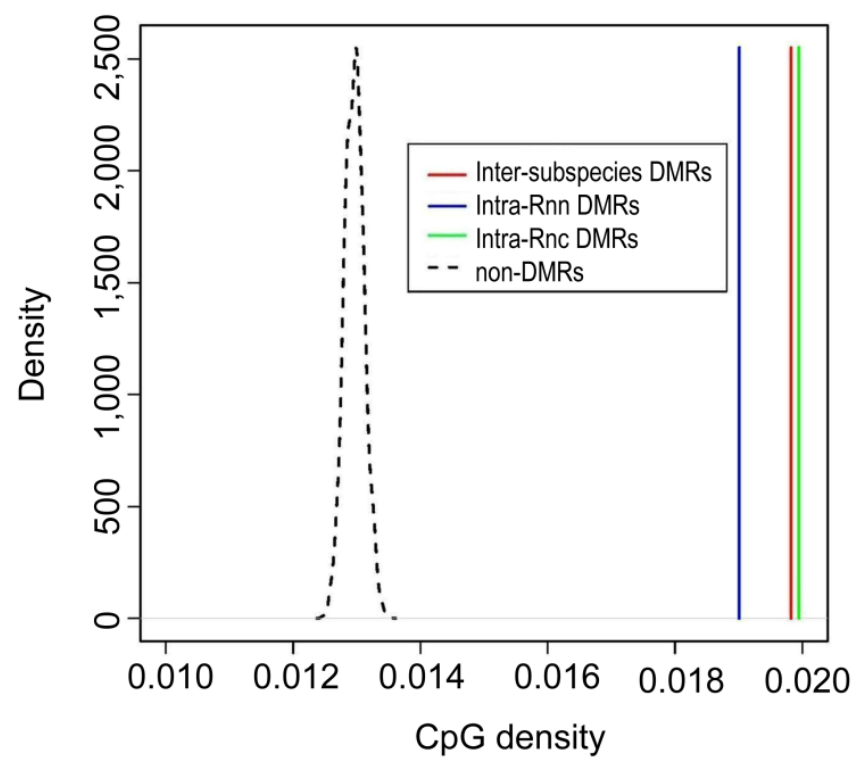

Supplemental figure 6. Comparison of CpG density. CpG density was calculated using the combined length of DMRs and corresponding non-DMRs using the second sampling method. 